\title{
Efeitos não Lineares em Fibras com Aplicação em Sensoriamento Distribuído de Temperatura
}

\author{
Luís C. B. Silva ${ }^{1}$, Maria J. Pontes ${ }^{2}$, Marcelo E. V. Segatto ${ }^{3}$ \\ Departamento de Engenharia Elétrica, Universidade Federal do Espírito Santo, Vitória, ES - Brasil \\ bluiscicero@yahoo.com ${ }^{1}$,mjpontes@ele.ufes.br ${ }^{2}$, segatto@ele.ufes.br ${ }^{3}$
}

\begin{abstract}
Resumo
Em projetos de sistemas de transmissão via fibras ópticas, deve-se atender critérios de operação que permitam a propagação da luz na fibra. Pode-se citar, por exemplo, as penalidades de potência como os mecanismos que degradam o sinal óptico à medida que o mesmo se propaga. Nesse contexto, parece ser intuitivo que para superar tais penalidades, basta manter a potência de entrada tão alta quanto possível. Entretanto, isto não deve ocorrer, pois a alta densidade de potência no núcleo da fibra ativa seu comportamento não linear, ocasionando no surgimento de efeitos não lineares que degradam o sinal de informação. Nessa perspectiva, o estudo aqui proposto tem por finalidade mostrar que embora efeitos não lineares não sejam desejáveis quando se pretende transmitir informação em fibras ópticas, os mesmos mostram-se benéficos quando se almeja utilizar a própria fibra como meio sensor, por exemplo, para interrogação distribuída de temperatura.
\end{abstract}

Resumo: Efeitos não lineares, interrogação de temperatura, fibras ópticas.

\begin{abstract}
In transmission systems projects via optical fibers must meet operational criteria that allow the light propagation in fiber. One can mention, for example, the power penalty as mechanisms that degrade the optical signal as it propagates into the fiber. Just increasing the input power to overcome loss effects in a fiber optic link is not effective to prevent attenuation. Instead, it can cause power penalties. The high power density in the fiber core activates its non-linear behavior. As a result, coupling high levels of light intensity into the fiber rises nonlinear effects that degrade the information signal. This paper discusses that although non-linear effects are undesirable when one wishes to transmit information in optical fibers, they prove to be beneficial if one wishes to use the fiber itself as a sensor means, for example, for the interrogation distributed temperature .
\end{abstract}

Keywords: Non-linear effects, interrogation temperature, optical fibers. 


\section{Blucher Proceedings \\ VII Encontro Científico de Física Aplicada Blucher}

\section{Introdução}

Sistemas modernos de comunicações com enlaces de grandes comprimentos, exigem altos valores de potência em um mesmo comprimento de onda. Isto faz com que a densidade de potência seja suficientemente elevada para forçar a fibra a operar em regiões não lineares [1].

Por conseguinte, trabalhos recentes abordam como efeitos não lineares (ENL) degradam o sinal de informação e como os mesmos podem ser reduzidos [2, 3, 4]. Todavia, estudos explorando tais não linearidades em fibras, para aplicação em sensoriamento, compõem atualmente um intenso campo de pesquisa na área de comunicações ópticas. Estes sensores mostram-se bastante promissores por vários motivos, entre eles, pode-se destacar o potencial comercial desta tecnologia, na exploração de diversos parâmetros físicos como temperatura, pressão, tensão, vibração, corrente elétrica, entre outros [5, 6, 7, 8].

Logo, o objetivo deste trabalho é abordar aspectos relacionados à natureza dos ENL, e assim, compreender como estes podem ser utilizados para sensoriar temperatura ao longo de um cabo de fibra óptica. A abordagem considera as equaçoes de Maxwell, uma vez que, princípios de óptica geométrica tornam-se limitados para tal análise. Esta permitirá compreender os fenômenos de geração de luz, emissão e absorção de energia por átomos e moléculas, bem como as interações com o meio e partículas carregadas.

Assim sendo, o artigo será apresentado da seguinte forma: $\mathrm{Na}$ Seção 2 discute-se a natureza dos ENL em fibras ópticas. Na Seção 3 é apresentada como obter a informação de temperatura a partir desses efeitos. Por último, as conclusões são apresentadas na Seção 4.

\section{Efeitos não Lineares em Fibras Ópticas}

A maioria dos assuntos tratados em comunicações ópticas, refere-se a óptica linear. Sendo a propagação de ondas luminosas descrita por equações de ondas lineares, que obedecem ao princípio da superposição. Entretanto, da mesma forma que em sistema mecânico simples como um objeto preso a uma mola pode ter uma resposta não linear quando sujeito a forças intensas, é razoável supor que um campo óptico elevado, propagando-se na fibra evidencie não linearidades.

Contudo, o domínio da óptica não linear é extenso, contemplando vários fenômenos para além dos referidos neste artigo. Por consequência, a abordagem aqui descrita, se restringe em apresentar de que maneira obter a informação de temperatura ao longo da fibra óptica, a partir de um sinal propagando-se na mesma, e cuja origem é nos ENL que podem sob determinadas circunstâncias serem gerados.

De fato, a observação e estudo dos ENL em fibras tornou-se possível com o advento de fontes lasers de alta intensidade e coerentes [9, 10], pois para altas densidades de potência no núcleo da fibra, a mesma pode ser considerada um meio não linear.

Em princípio, com a finalidade de melhor compreender como tais não linearidades surgem, pode-se considerar o meio de propagação como um conjunto de osciladores harmônicos forçados, ou seja, átomos com elétrons ligados pelo campo eletromagnético. Uma vez que osciladores reais apresentam comportamento não linear para grandes amplitudes de oscilação [11].

Neste contexto, a propagação da luz 


\section{Blucher Proceedings VII Encontro Científico de Física Aplicada Blucher}

em um meio material assume ter uma relação linear entre o campo eletromagnético e a resposta desse meio, i.e., a polarização é dada por [10]:

$$
P=\varepsilon_{0} \chi E
$$

Onde $\chi$ é a susceptibilidade do meio, e $\varepsilon_{0}$ a permissividade dielétrica do vácuo. Por outro lado, quando o meio é não linear, temos [10]:

$$
P=\varepsilon_{0} \chi_{1} E+\varepsilon_{0}\left(\chi_{2} E^{2}+\chi_{3} E^{3}+\ldots\right)
$$

O primeiro termo no membro direito em (2) corresponde à parte linear da polarização, enquanto que o segundo termo corresponde à parte não linear. Sendo o termo de terceira ordem da susceptibilidade o responsável por dar origem as não linearidades na fibra.

Portanto, quando luz de alta intensidade interage com as moléculas que constituem o material da fibra óptica, ocorrem trocas de energia entre os fótons da luz incidente e as moléculas do material (geralmente sílica fundida). Essas trocas de energia geram novos campos eletromagnéticos com frequências diferentes que se propagam em todas as direções [12]. Caso a frequência da radiação espalhada for menor que a frequência da radiação incidente, o processo de espalhamento absorve energia. Isto implica dizer que as moléculas do meio absorveram energia dos fótons da luz incidente, produzindo fótons espalhados com menor energia. Esse espalhamento é denominado Stokes [13].

Por outro lado, se a radiação espalhada tiver frequência maior que a da radiação incidente, as moléculas do meio cederam parte de sua energia para os fótons da luz incidente. Isto faz com que sejam gerados fótons com maior energia. Esse espalhamento recebe o nome de Anti-Stokes [13].

Neste ponto, fica claro que a geração de fótons Anti-Stokes é menor do que fótons Stokes pelo fato da probabilidade de encontrar moléculas em estados de maior energia, ser inferior a probalibidade de encontrá-las em seus estados fundamentais de energia.

Considerando ainda, a geometria da fibra óptica e seus vários modos de propagação, a interpretação de como a luz é guiada deve ser realizada a partir das equações de Maxwell, incluindo as condições de contorno na interface do núcleo com a casca [1]. Tal abordagem, permite prever que uma pequena parcela dos fótons Stokes e Anti-Stokes espalhados serão capturados e guiados ao longo da fibra. Isto implica, no surgimento das componentes Stokes e Anti-Stokes, que podem propagar-se tanto no sentido copropagante como contra propagante.

Além disso, levando em consideração o fato de que o meio material de propagação (neste caso a fibra óptica) pode ter suas propriedades microscópicas alteradas (em pontos específicos) por fatores externos como a temperatura por exemplo, torna-se claro a possibilidade de se avaliar as consequências destes fatores sobre a propagação das componentes Stokes e Anti-Stokes, uma vez que, tais componentes são oriundas da interação da luz com a estrutura da fibra.

Portanto, a possibilidade de medir os fatores externos, que agem sobre o meio de propagação, através da caracterização de ENL, fornece o princípio geral para a pesquisa de sensores ópticos distribuídos [14]. 


\section{Blucher Proceedings VII Encontro Científico de Física Aplicada Blucher}

\section{Extraindo a informa- ção da Temperatura}

Admitindo-se que sejam satisfeitas as condições para gerar não linearidades, e dependendo da potência acoplada na fibra, diversos efeitos não lineares ocorrerão simultaneamente, tais como espalhamento Raman, Brillouin e Rayleigh [12, 10]. Conforme ilustra a Figura 1.

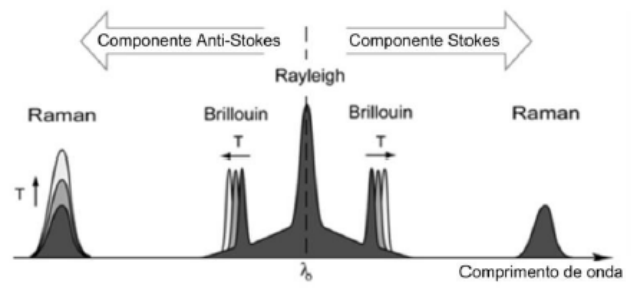

Figura 1. Espectro do comprimento de onda do espalhamento Rayleigh, Raman e Brillouin [15].

Nota-se, por meio da Figura 1, que para o caso da temperatura, a mesma afeta diretamente a intensidade do sinal Raman e a frequência do sinal Brillouin.

A escolha destes efeitos para sensoriamento dependerá do parâmetro físico a ser medido; do comprimento do elemento sensor; da sensibilidade do efeito com relação ao parâmetro; ou ainda do objetivo do sistema sensor, que cada não linearidade oferece [13].

Ocorrendo então, variações de temperatura em pontos específicos na fibra, as moléculas do meio nestes pontos irão absorver parte dessa energia térmica, passando para um estado de maior energia (energia vibracional). Nestas regiões, como descrito na Seção anterior, ocorrerá maior geração de fótons Anti-Stokes.

Desse modo, se o parâmetro físico que deseja-se medir é a temperatura na fibra, e a mesma não for constante, os fótons AntiStokes terão energias diferentes. Consequentemente, a componente Anti-Stokes gerada será modulada pelas alterações na temperatura. Desta forma, medindo esta modu- lação é possível obter o perfil de temperatura ao longo de toda a extensão da fibra [12].

Algumas ressalvas na obtenção da temperatura devem ser feitas. Primeiramente, as componentes Stokes e Anti-Stokes sofrem atenuação ao longo de sua propagação, e como esta depende da frequência dos campos ópticos, as potências detectadas precisam ser corrigidas antes de traçar o perfil de temperatura [14, 16, 17]. Segundo, somente a componente Anti-Stokes possui dependência com a temperatura, conforme ilustra a Figura 2.



Figura 2. Dependência da componente AntiStokes com a temperatura.

Entretanto, geralmente usa-se também a componente Stokes [18] (ou Rayleigh [19]), a fim de normalizar a componente Anti-Stokes. Isto permite cancelar os efeitos provocados pela atenuação e perdas locais, que podem afetar a medida da temperatura . Porém, pode-se interragar a temperatura medindo somente a componente Anti-Stokes [20].

Logo após obtida a informação de temperatura, faz-se necessário localizar em que região da fibra tal medida corresponde. Este procedimento se dá utilizando o tempo entre o lançamento do pulso de luz acoplado à fibra, e o retorno das ondas espalhadas (detecta-se normalmente as componentes no sentido contra-propagante) [14]. Portanto, considerando $\triangle t$ o intervalo de tempo entre o momento do lançamento do pulso de estímulo e o momento do retorno dos campos excitados, pode-se mostrar que o espalhamento gerado é referente à posição $z$, tal que [14]:

$$
z=\frac{c}{2 n} \triangle t
$$




\section{Blucher Proceedings VII Encontro Científico de Física Aplicada Blucher}

Sendo $c$ e $n$, a velocidade da luz no vácuo, e o índice de refração do núcleo da fibra, respectivamente.

\section{Conclusão}

As fibras ópticas apresentam uma variedade de efeitos não lineares que não são desejáveis pelos projetistas de sistemas de telecomunicações por afetarem o desempenho do sistema de formas adversas. Entretanto, o tratamento adequado de tais não linearidades permite desenvolver dispositivos, como por exemplo sensores, utilizando a própria fibra como elemento sensor, permitindo medir parâmetros físicos como temperatura, pressão, corrente elétrica, entre outros.

Portanto, do tratamento descrito neste trabalho, aspectos relacionados a ocorrência de efeitos não lineares em fibras ópticas foram levantados, como também, de qual maneira tal conhecimento possibilita obter a informação de temperatura em fibras ópticas.

\section{Referências}

[1] J. A. J. Ribeiro, "Características da propagação em fibras ópticas," Instituto $\mathrm{Na}$ cional de Telecomunicações, 1999.

[2] O. F. Pereira et al., "Caracterização de fibras ópticasa altamente não lineares," 2012.

[3] T. Oliveira, R. Plácido, A. Mendes, and E. Silva, "Os efeitos não lineares em sistemas de comunicações ópticas." Communication Technology, 2011.

[4] Y. Zou, O. Raeesi, L. Antilla, A. Hakkarainen, J. Vieira, F. Tufvesson, Q. Cui, and M. Valkama, "Impact of power amplifier nonlinearities in multi-user massive mimo downlink," in 2015 IEEE Globecom Workshops (GC Wkshps). IEEE, 2015, pp. 1-7.
[5] J. Bazzo, D. Pipa, C. Martelli, E. Silva, and J. C. C. da Silva, "Improving spatial resolution of raman dts using total variation deconvolution," IEEE Sensors Journal, vol. PP, no. 99, pp. 1-1, 2016.

[6] H. S. Pradhan and P. K. Sahu, "Characterisation of raman distributed temperature sensor using deconvolution algorithms," Optoelectronics, IET, vol. 9, no. 2, pp. 101-107, 2015.

[7] E. Udd and W. Spillman, "The emergence of fiber optic sensor technology," Fiber Optic Sensors: An Introduction for Engineers and Scientists; Udd, E., Ed, pp. 1-8, 2011.

[8] F. Marignetti, E. de Santis, S. Avino, G. Tomassi, A. Giorgini, P. Malara, P. D. Natale, and G. Gagliardi, "Fiber bragg grating sensor for electric field measurement in the end windings of high-voltage electric machines," IEEE Transactions on Industrial Electronics, vol. 63, no. 5, pp. 2796-2802, May 2016.

[9] R. W. Boyd, Nonlinear Optics. Academic Press, USA, 2003.

[10] G. P. Agrawal, Nonlinear Fiber Optics. Academic Press, USA, 2007.

[11] Y.-R. Shen, "Principles of nonlinear optics," 1984.

[12] X. Bao and L. Chen, "Recent progress in distributed fiber optic sensors," Sensors, vol. 12, no. 7, pp. 8601-8639, 2012.

[13] A. D. G. Rodrigues and J. C. Galzerani, "Espectroscopias de infravermelho, raman e de fotoluminescência: potencialidades e complementaridades," Revista Brasileira de Ensino de Fisica, vol. 34, no. 4, p. 4309, 2012.

[14] J. F. Rossetto and E. C. Ferreira, "Sensores distribuídos utilizando efeitos não-lineares em fibras ópticas para 


\section{Blucher Proceedings VII Encontro Científico de Física Aplicada Blucher}

a aplicação em estruturas inteligentes," FEEC/UNICAMP, 2004.

[15] D. Inaudi and B. Glisic, "Integration of distributed strain and temperature sensors in composite coiled tubing," in Smart Structures and Materials. International Society for Optics and Photonics, 2006, pp. 616717-616717.

[16] E. Karamehmedovic, "Incoherent optical frequency domain reflectometry for distributed thermal sensing," Technical University of Denmark, Department of Communications, Optics and Materials, 2006.

[17] M. A. Farahani and T. Gogolla, "Spontaneous raman scattering in optical fibers with modulated probe light for distributed temperature raman remote sensing," Journal of Lightwave Technology, vol. 17, no. 8, p. 1379, 1999.
[18] B. Culshaw and A. Kersey, "Fiber-optic sensing: A historical perspective," Journal of lightwave technology, vol. 26, no. 9, pp. 1064-1078, 2008.

[19] G. Bolognini, J. Park, M. A. Soto, N. Park, and F. Di Pasquale, "Analysis of distributed temperature sensing based on raman scattering using otdr coding and discrete raman amplification," Measurement Science and Technology, vol. 18, no. 10, p. 3211, 2007.

[20] M. A. Soto, A. Signorini, T. Nannipieri, S. Faralli, and G. Bolognini, "Highperformance raman-based distributed fiber-optic sensing under a loop scheme using anti-stokes light only," IEEE Photonics technology letters, vol. 23, no. EPFL-ARTICLE-182043, pp. 534-536, 2011. 